\title{
Conhecimento sobre Suplementos Alimentares por Profissionais da Saúde
}

\author{
Knowledge about Food Supplements by Health Professionals
}

\section{RESUMO}

Objetivo: Analisar o consumo e o conhecimento dos profissionais da área da saúde sobre os suplementos alimentares. Metodologia: A amostra foi composta por 30 indivíduos sendo 15 educadores físicos e 15 nutricionistas que atuam em academias e clínicas em uma cidade da região norte do Brasil. Para coleta de dados foi realizado uma entrevista semiestruturada. Resultados: Os participantes da pesquisa foram, em geral, jovens adultos de ambos os sexos, com formação acadêmica com menos de 10 anos. Estes reconheciam que estes produtos podem apresentar efeitos colaterais, porém, em $90 \%$ dos casos nunca tinham presenciado tais efeitos nos pacientes. Os resultados demonstraram uma alta porcentagem de profissionais que indicavam a utilização de suplementos. Os mais indicados foram os carboidratos, aminoácidos e bebidas isotônicas. Para as questões referentes ao conhecimento sobre a ação e finalidade dos SA, tanto os nutricionistas quanto os educadores físicos tiveram uma média de acerto de aproximadamente $70 \%$. Este resultado mediano pode ser reflexo da proximidade entre a atuação profissional e o período de formação destes profissionais. Conclusão: Conclui-se que o conhecimento dos profissionais foi mediano, poucos observaram em seus pacientes o surgimento de efeitos colaterais e a maioria não tinha formação, em nível de pósgraduação, na área das ciências do esporte.

\section{DESCRITORES}

Suplementos Alimentares. Nutricionistas. Educação Física. Treinamento.

\begin{abstract}
Objective: The objective of this study was to analyze the consumption and knowledge of health professionals about dietary supplements. Methodology: The sample consisted of 30 individuals, 15 physical educators and 15 nutritionists who work in gyms and clinics in a city in the northern region of Brazil. For data collection a semi-structured interview was conducted. Results: The research participants were in general young adults of both sexes, with academic training under 10 years. They recognized that these products can have side effects, but in $90 \%$ of cases they had never seen such effects in patients. The results showed a high percentage of professionals indicating the use of supplements. The most indicated were carbohydrates, amino acids, and isotonic drinks. For questions related to the knowledge about the action and purpose of SA, both nutritionists and physical educators had an average of approximately $70 \%$ of accuracy. This median result may reflect the proximity between the professional performance and the training period of these professionals. Conclusion: We can conclude from this study that the knowledge of the professionals was medium, few observed in their patients the appearance of side effects and most had no training at the postgraduate level in the field of sports sciences.
\end{abstract}

\section{DESCRIPTORS}

Dietary Supplements. Nutritionists. Physical Education.Training.

${ }^{1}$ Nutricionista, graduada pela Universidade Federal do Tocantins, especialista em Saúde da Família e Comunidade. Nutricionista do Núcleo de Apoio à Saúde da Família (NASF) da Secretaria Municipal de Saúde de Palmas-TO.

${ }^{2}$ Nutricionista, graduada pela Universidade Federal do Tocantins. Nutricionista do Núcleo de Apoio à Saúde da Família (NASF) da Secretaria Municipal de Saúde de Palmas-TO.

${ }^{3}$ Professora convidada na Universidade Federal do Tocantins. Nutricionista da Secretaria Municipal de Saúde de Palmas-TO.

${ }^{4}$ Professor do Curso de Nutrição e dos Programas de Mestrado em Ciências da Saúde e Mestrado em Ciência e Tecnologia de Alimentos da Universidade Federal do Tocantins. 
A busca por um estilo de vida saudável aliado a melhoria estética, tem levado uma parcela da população a prática de atividade física ${ }^{1}$. Segundo a Associação Brasileira de Academias $^{2}$ o número de academias no Brasil passou de 15.000 no ano 2010 para mais de 30.000 estabelecimentos em 2014, atendendo a um público de cerca de 8 milhões de alunos, com faturamento de mais de $R \$ 2,5$ bilhões de reais.

Além da prática de exercícios físicos, é observado na população a mudança nos hábitos alimentares ${ }^{3,4}$; a maior procura por cirurgias plásticas e o elevado consumo de suplementos alimentares $(\mathrm{SA})^{5}$. Estes últimos são comercializados e propagados muitas vezes com a promessa de resultados rápidos, contudo, o consumo sem a devida orientação, e sem levar em consideração o biotipo do indivíduo, as carências nutricionais, os exames físicos e bioquímicos adequados, podem levar a maior probabilidade de ocorrência de efeitos adversos ou até intoxicações ${ }^{6,7}$.

A devida orientação deve ser feita por profissional especialista em nutrição esportiva, porém, é sabido que a população recorre a diferentes fontes para obter informações, entres estes a internet, familiares, instrutores e educadores físicos em academias ${ }^{8,9}$. Vale ressaltar que este último está em contato por maiores períodos de tempo com os praticantes de atividade física.

Portanto, é imperativo que o profissional da área da saúde ao auxiliar esta população ou orientá-la, no caso dos nutricionistas, tenha formação específica em ciências do esporte e conhecimentos sobre suplementos alimentares, para propiciar ao paciente os efeitos desejados e minimizar a probabilidade do aparecimento de efeitos adversos, tais como problemas hepáticos, aumento de ácido úrico plasmático, problemas renais entre outros $^{10,11 .}$.

Portanto, o objetivo deste estudo foi analisar o uso e o conhecimento dos profissionais da área da saúde, nutricionistas e educadores físicos, sobre suplementos alimentares.

\section{METODOLOGIA}

Foi realizado um estudo transversal analítico-descritivo, com uma amostra composta por 30 profissionais da área da saúde, sendo 15 educadores físicos e 15 nutricionistas de ambos os sexos, atuantes em academias de ginástica ou clínicas na Cidade de Palmas - Tocantis, Brasil, selecionados de forma aleatória.

Os profissionais foram abordados em seu ambiente de trabalho e, após a explanação sobre os objetivos da pesquisa, os indivíduos foram convidados a assinar um Termo de consentimento livre e esclarecido, seguindo as normas da resolução específica do Conselho Nacional de Saúde ${ }^{12}$. Então, foi realizada uma entrevista semiestruturada. Previamente, foi realizado um estudo-piloto com 10 indivíduos, para verificar a viabilidade e os possíveis problemas relacionados à metodologia e aos instrumento utilizados.

O questionário foi composto por três etapas: i) aspectos socioculturais; ii) perguntas sobre à orientação, consumo e efeitos adversos relacionados aos suplementos alimentares e iii) teste onde o indivíduo deveria relacionar o tipo de suplemento com sua respectiva função. 
Os diferentes tipos de suplementos alimentares foram agrupados segundo Oliveira e colaboradores ${ }^{13}$ : i) Aminoácidos ou outros concentrados protéicos (Whey protein, BCAA, Albumina); ii) Vitaminas ou complexos vitamínicos (Cebion, Provit); iii) Carboidratos (Dextrina, Maltose); iv) Creatina; v) L-Carnitina; vi) Bebida isotônica (Gatorade); e vii) Produtos fitoterápicos (guaraná, ginseng).

Os dados obtidos foram agrupados e analisados por meio de estatística descritiva (frequências relativas, média e desvio-padrão), além de ANOVA e do teste de Tukey, ao nível de significância de $p<0,05$. Foi utilizado para estas análises o software GraphPad Prism 6.0.

\section{RESULTADOS}

Neste estudo, os profissionais entrevistados tinham faixa etária entre 20 a 40 anos de idade (Tabela 1), um pouco mais de $50 \%$ foi do gênero feminino, com nível

Tabela 1. Aspetos socioculturais dos profissionais educadores físicos e nutricionistas

\begin{tabular}{|c|c|c|}
\hline & $\begin{array}{l}\text { Porcentagem } \\
\text { absoluta }(n=30)\end{array}$ & $\begin{array}{c}\text { Porcentagem } \\
\text { relativa }(\%)\end{array}$ \\
\hline \multicolumn{3}{|l|}{ Faixa etária (anos) } \\
\hline $20-30$ & 15 & 50 \\
\hline $31-40$ & 15 & 50 \\
\hline $41-50$ & - & - \\
\hline$>50$ & - & - \\
\hline \multicolumn{3}{|l|}{ Gênero } \\
\hline Mulheres & 16 & 53 \\
\hline Homens & 14 & 47 \\
\hline \multicolumn{3}{|l|}{ Escolaridade } \\
\hline Graduação & 13 & 43 \\
\hline Especialização & 14 & 47 \\
\hline Mestrado & 02 & 07 \\
\hline Doutorado & 01 & 03 \\
\hline \multicolumn{3}{|c|}{ Renda familiar (salário-mínimo) } \\
\hline$<1$ & - & - \\
\hline 1 a 4 & 03 & 10 \\
\hline$>4$ & 27 & 90 \\
\hline \multicolumn{3}{|c|}{ Atividade física em que atua } \\
\hline Musculação & 15 & 46 \\
\hline Natação & 01 & 03 \\
\hline Artes marciais & 01 & 03 \\
\hline Outros & 16 & 49 \\
\hline \multicolumn{3}{|l|}{ Tempo de atuação (anos) } \\
\hline$<1$ & - & - \\
\hline 1 a 4 & 09 & 30 \\
\hline 5 a 7 & 08 & 27 \\
\hline 8 a 10 & 07 & 23 \\
\hline$>10$ & 06 & 20 \\
\hline
\end{tabular}


de escolaridade majoritária de especialistas $(47 \%)$ e graduados (43\%), com renda familiar acima de 4 salários mínimos em $90 \%$ dos casos (Tabela 1). A principal atividade física na qual estes profissionais atuavam foi a musculação (46\%) e, apenas, $20 \%$ atuavam profissionalmente há mais de 10 anos (Tabela 1).

Do total de entrevistados, $70 \%$ indicou ter conhecimento de que os suplementos alimentares podem apresentar efeitos colaterais, porém, 90\% relataram nunca ter observado tais efeitos (Figura 1) e dentre os suplementos mais indicados pelos mesmos estão os carboidratos, aminoácidos e os isotônicos.

Para as questões referentes às funções e indicações dos suplementos alimentares,

Figura 1. Porcentagem de respostas de efeito colateral pelo uso de suplementos alimentares, em pacientes/clientes após orientação e tipo de suplemento indicado

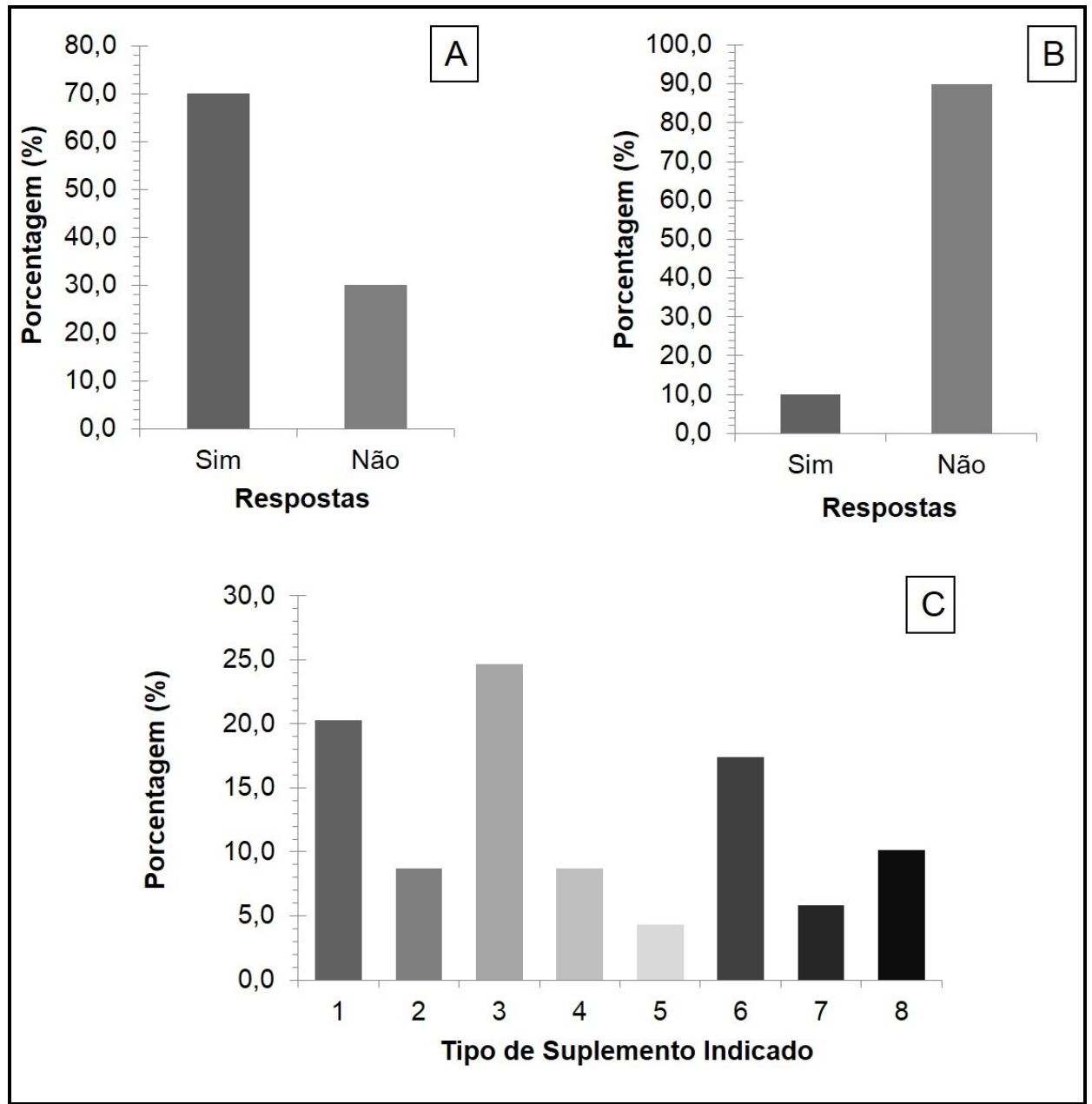

A) Conhecimento de efeito colateral pelo uso de suplementos alimentares; B) Observação de efeito colateral em pacientes/clientes após orientação; C) Tipo de suplemento indicado: 1) Aminoácido; 2) Complexo Vitamínico; 3) Carboidrato; 4) Creatina; 5) L-Carnitina; 6) Isotônico; 7) Fitoterápicos; 8) Outros. 
nutricionistas e educadores físicos tiveram porcentagem de acertos das respostas semelhantes, em torno de $70 \%$ (Figura 2), sendo que a maior porcentagem de acertos foi verificada para os produtos: complexo vitamínico, isotônico e fitoterápicos (Figura 3).

Figura 2. Porcentagem de acertos e erros nas questões referentes ao conhecimento sobre suplemento alimentar e sua função

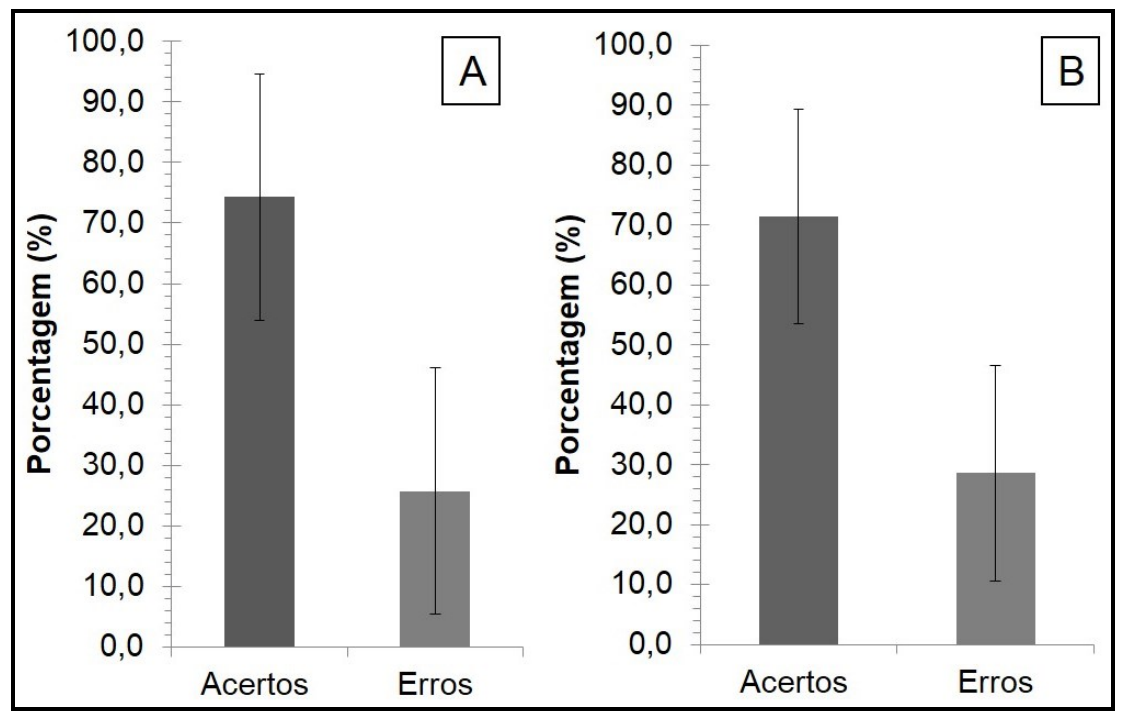

A) Educadores Físicos; B) Nutricionistas

Figura 3. Acertos por alternativas das questões referentes ao conhecimento sobre suplementos alimentares

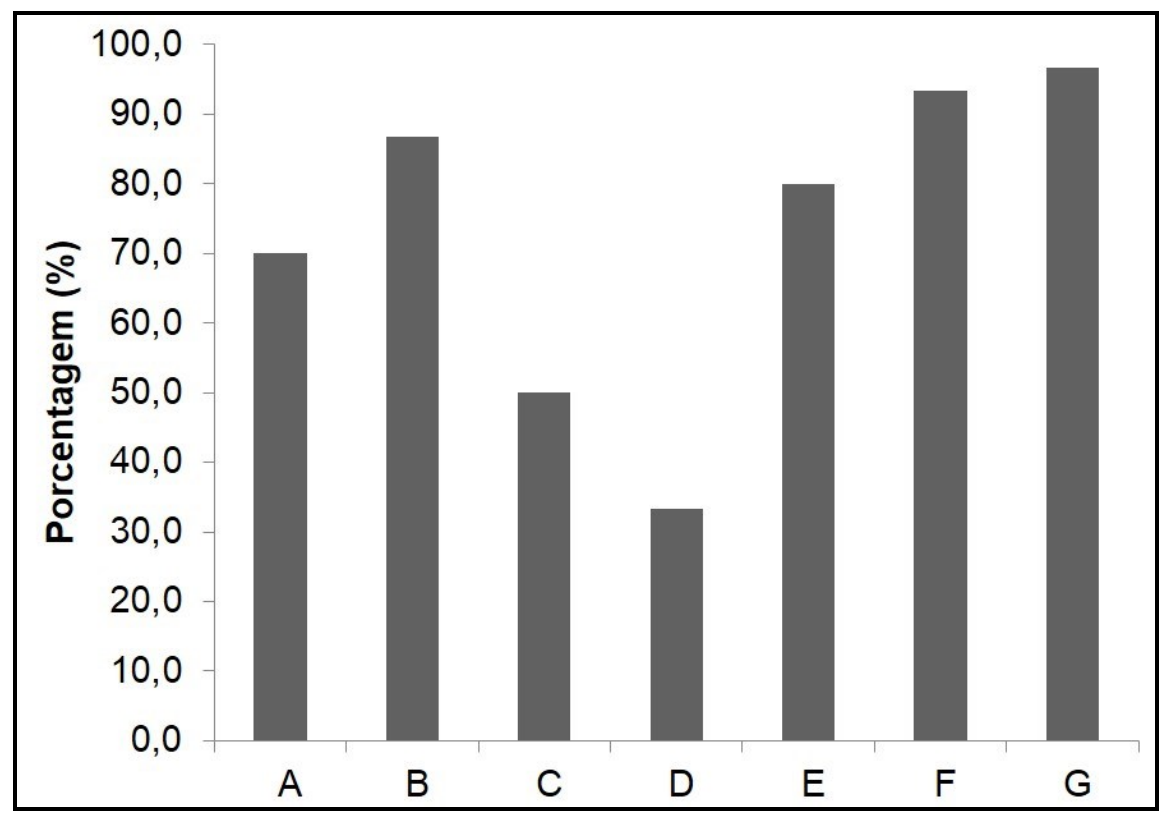

A) Aminoácidos; B) Complexo Vitamínico; C) Carboidrato; D) Creatina; E) LCarnitina; F) Isotônico; G) Fitoterápicos. 


\section{DISCUSSÃO}

O perfil dos entrevistados, de jovens adultos, de ambos os sexos, reflete em parte o perfil da população da Cidade de Palmas ${ }^{14}$, por ser esta uma cidade nova que atraiu e ainda atrai pessoas jovens atrás de estudo e oportunidades no mercado. Pela formação recente dos profissionais entrevistados também pode ser devido à característica desta capital, que tem apenas 29 anos.

Os resultados demonstraram uma porcentagem de $70 \%$ dos profissionais educadores físicos e nutricionistas que indicavam a utilização de SA, o que pode ser um reflexo da maior demanda da população por estas substâncias, o que resulta em maior procura de informações junto a estes profissionais. O incentivo ao uso de suplementos está presente em propagandas, internet e, alguns estudos têm demonstrado que os usuários destes produtos de venda livre, o faz mediante, muitas vezes, por indicação de instrutores e treinadores sem habilitação técnica e ética para o mesmo ${ }^{15,16}$.

A devida orientação e a prescrição devem ser feitas em clínicas, por nutricionistas e médicos, para o diagnóstico da real necessidade de utilização destas substâncias, que podem provocar efeitos adversos, desde leves, como câimbras e fadiga até problemas graves como insuficiência renal ${ }^{17}$.

Do total de entrevistados, $70 \%$ responderam conhecer que os SA podem causar efeitos adversos (Figura 1A), porém, $90 \%$ nunca presenciaram tais efeitos em seus pacientes/clientes (Figura1B). Os SA mais indicados foram os carboidratos $(24,6 \%)$, aminoácidos $(20,3 \%)$ e bebidas isotônicas
$(17,4 \%)$ (Figura1C). A alta porcentagem de indicação pode ser reflexo do mercado e a população que tem consumido cada vez mais estas substâncias. Entretanto, a alta porcentagem de não observação de efeitos colaterais pode ser um dado preocupante, pois normalmente, associasse com o não acompanhamento dos pacientes após o uso dos SA.

Para as questões referentes ao conhecimento sobre a ação e a finalidade dos SA, tanto os nutricionistas quanto os educadores físicos tiveram uma média de acerto de cerca de $70 \%$ (Figura 2), não tendo diferença significativa quando comparado os dois grupos. Portanto, é imperativo ainda salientar que os profissionais nutricionistas são prescritores para estas substâncias.

Quanto à porcentagem de acerto por questões na avaliação, foi observado maior índice de acerto para os fitoterápicos, bebidas isotônicas e vitaminas (Figura 3) e, menor nível de acerto, para os carboidratos e a creatina (Figura 3).

Esta análise demonstra em qual tipo de SA existe maior necessidade de intervenção e este resultado pode ser preocupante se observado que os carboidratos foram os SA mais indicados (Figura1C). Deve-se levar em consideração que $43 \%$ da amostra tinha apenas a graduação e, muitos que tinham especialização, não trabalhavam exclusivamente nesta área.

Segundo Fayh e colaboradores ${ }^{18} \mathrm{o}$ apelo do marketing tem gerado a disseminação e a popularização destes produtos, o que leva ao uso indevido tanto em termos de quantidades e de tempo de uso. Aliado a classificação de algumas destas substâncias 
como alimento pela ANVISA, seu comercio é realizado livremente ${ }^{18}$. Logo, uma união de fatores tem implicado na dificuldade e na orientação quanto ao uso de suplementos alimentares.

Outra questão a ser enfatizada é a distinta formação entre os prescritores ${ }^{19}$, a necessidade de várias habilidades por partes desse profissional que irá atuar na área esportica ${ }^{20} \mathrm{e}$, por fim, em muitos cursos a abordagem da temática esportiva é reduzida. Pode-se, então, hipotetizar que existe uma necessidade de formação especifica para estes profissionais, com foco maior para os nutricionistas, uma vez que trabalham diretamente com a prescrição, ao avaliar os resultados obtidos e o tempo de formação destes profissionais estudados. Novos estudos sobre a origem da formação e das

\section{REFERÊNCIAS}

1. Cheffer NM, Benetti F. Análise do consumo de suplementos alimentares e percepção corporal de praticantes de exercícios físicos em academia do município de Palmitinho-RS. Rev Bras de Nutrição Esportiva. 2016; 10(58): 390-401.

2. ACAD - Associação Brasileira de Academias. Mercado.2017.

3. Cattafesta M, Zandonade E, Bissoli NS, Salaroli LB. Padrões alimentares de trabalhadores bancários e sua associação com fatores socioeconômicos, comportamentais e laborais. Ciência \& Saúde Coletiva. 2019; 24(10):3909-3922.

4. Santos GMGC, Silva AMR, Carvalho WO, Rech CR. Loch $M R$. Barreiras percebidas para o consumo de frutas e de verduras ou legumes em adultos brasileiros. Ciência \& Saúde Coletiva. 2019; 24(7):2461-2470.

5. Sá VM, Carvalho MA, Guedes AC, Santos JB, Sá LM, Gratão LHA, Nascimento GN. Estado nutricional e uso de suplementos alimentares por acadêmicos de nutrição da Universidade Federal do Tocantins. Rev Bras Nutr Esport. 2018; 12(74):724-732. bases curriculares dos mesmos podem ser relevantes para melhor entender e auxiliar nas atividades destes profissionais.

\section{CONCLUSÃO}

Podemos concluir com este trabalho que o conhecimento dos profissionais foi mediano, sendo que poucos observaram em seus pacientes o surgimento de efeitos colaterais e a maioria não tinha formação em nível de pós-graduação, na área das ciências do esporte.

\section{AGRADECIMENTOS}

Agradecemos a Universidade Federal do Tocantins e ao CNPq pelo apoio a realização desta pesquisa.

6. Ronis MJJ, Pedersen KB, James Watt J. Adverse effects of nutraceuticals and dietary supplements. Annu Rev Pharmacol Toxicol. 2018; 06(58):583-601.

7. Knapik JJ, Trone DW, Austin KG, Steelman RA, Farina EK, Lieberman HR. Prevalence, adverse events, and factors associated with dietary supplement and nutritional supplement use by us navy and marine corps personnel. J Acad Nutrition Dietetics. 2016; 116(9): 1423-1442.

8. Souza IR, Cargnin-Carvalho A. Consumo de suplementos nutricionais nas academias da cidade de Braço do Norte. Rev Bras Nutr Esport. 2018; 12(70): 213-221.

9. Ana Catarina Gomes AC, Figueiredo SM, Souza AA Avaliação do consumo de suplementos por praticantes de musculação em academias de Ouro Preto - MG Demetra. 2018; 13(4): 937-951.

10. Cava TA, Madruga SW, Teixeira GDT, Reichert FF, Silva MC, Rombaldi AJ. Consumo excessivo de suplementos nutricionais entre profissionais atuantes em academias de ginástica de Pelotas, Rio Grande do Sul, 2012. Epidemiol Serv Saude. 2017; 26(1):99-108.

11. Carvalho JO, Oliveira BN, Machado AAN, Machado 
EP, Oliveira BN. Uso de suplementação alimentar na musculação: revisão integrativa da literatura brasileira. Conexões: Educ. Fís., Esporte e Saúde. 2018; 16(2):213225.

12. CNS - Conselho Nacional de Saúde: Comissão Nacional em Ética em Pesquisa. Resolução n466/12, 2012.

13. Oliveira SRU, Marin KA, Pereira RJ, Nascimento GNL. Avaliação do uso e conhecimento de suplementos alimentares por acadêmicos do curso de educação física. Revista Cereus. In press.

14. IBGE - Instituto Brasileiro de Geografia e Estatística. Cidades. 2017.

15. Maximiano CMBF, Santos LC. Consumo de suplementos por praticantes de atividade física em academias de ginástica da cidade de Sete Lagoas-MG. Rev Bras Nut Esport. 2017; 11(61):93-101.

16. Silva FR, Mendes AEP, Pinto FJM, Sampaio RMM, Morais VD, Brito FCR. Perfil nutricional e uso de suplementos alimentares: estudo com adultos praticantes de musculação. Motricidade. 2018; 14(1):271-278.

17. Sociedade Brasileira de Medicina do Esporte. Modificações dietéticas, reposição hídrica, suplementos alimentares e drogas: comprovação de ação ergogênica e potenciais riscos para saúde. Rev Bras Med Esp. 2009; 9(2):43-56.
18. Fayh APT, Silva CV, Jesus FRD, Costa GK. Consumo de suplementos nutricionais por frequentadores de academias da cidade de Porto Alegre. Rev Bras Ciênc Esp. 2013; 35:27-37.

19. Ferreira CC, Santos DM, Viebig RF, Frade RET. Atualidades sobre a suplementação nutricional com beta-alanina no esporte. Rev Bras Nutr Esp. 2015; 9(51):271-278.

20. Juzwiak CR. Reflection on sports nutrition: Where we come from, where we are, and where we are headed. Rev Nutr. 2016: 29(3): 435-444.

\section{CORRESPONDÊNCIA}

Guilherme Nobre L. do Nascimento

Laboratório de Ciências Básicas e da Saúde (LaCiBS). Avenida NS 15, 109 Norte - Plano Diretor Norte,

Universidade Federal do Tocantins,

Complexo de Laboratórios da Nutrição. CEP: 77001-090.

Palmas - Tocantins - Brasil.

E-mail: guilherme.nobre@uft.edu.br 